\title{
Susceptibility of anaerobic bacteria: implementation of cumulative antibiogram at hospital level
}

Milva Ballardini', Francesco Cortese' , Marina Cattivelli', Maria Letizia Schiavone', Luciano Spagnesi', Silvana Maiorano', Annunziata Tamburro', Marcello Meledandri'

I U.O.C. Microbiologia e virologia, Azienda Complesso Ospedaliero San Filippo Neri, Roma

2 U.O.C. Chirurgia d'urgenza, Azienda Complesso Ospedaliero San Filippo Neri, Roma

Key words: Anaerobes, Cumulative antibiogram, Gastrointestinal surgery

Realizzazione di un report locale di suscettibilità dei batteri anaerobi

\section{SUMMARY}

Empiric therapy should be modified according to local susceptibility data. This condition could not be satisfied for some pathogens, as anaerobes. Therefore, treatment of infections related to the anaerobic flora - e.g. gastrointestinal surgery - is based on presumptive ecology of site of the infection. The aim of the study was to arrange a local cumulative "antibiogram" to support empiric therapy of anaerobic infections, useful for surgical area.A systematic review of all bacterial identifications and susceptibility tests of anaerobic bacteria of inpatients, over eight year period, was performed. 255 non-duplicate strains were evaluated: 4I Gram positive and 214 Gram negative. Among genera, Bacteroides and Prevotella were the most represented. Over $75 \%$ of microorganisms derived from SSI specimens. It was observed high frequency of beta-lactamase producers ( 81\%). Chloramphenicol, imipenem, metronidazole, piperacillin-tazobactam (>95\% S) showed to be the most effective antimicrobials, followed by amoxicillin-clavulanate and cefoxitin (80-90\% S); clindamycin had poor effectiveness $(\sim 64 \%$ S). Our local data were similar with recognized European susceptibility of anaerobes.

\section{INTRODUZIONE}

Gli anaerobi costituiscono una componente non infrequente nelle infezioni postoperatorie, soprattutto dopo chirurgia del tratto gastrointestinale (6). Questi batteri rappresentano un rilevante problema clinico in quanto: a) peggiorano la prognosi, b) sono sottostimati dai laboratori per le difficoltà nella raccolta $\mathrm{e}$ nella lavorazione dei materiali associati (10). Quest'ultimo aspetto determina, come conseguenza clinico-terapeutica in ambito chirurgico, l'applicazione di terapie empiriche basate prevalentemente sulla conoscenza del microbiota intestinale $(3,12)$. Accanto a lavori che attribuiscono un ruolo importante all'indagine microbiologica, altri rimarcano il ruolo della terapia empirica, a prescindere dalle colture $(11,7)$. Le nuove linee guida sulle infezioni intra-addominali individuano gli spazi per l'indagine microbiologica, ma aumentano il valore prognostico dell'inquadramento clinico, dei fattori di rischio e della precocità della terapia (16).

I report di suscettibilità, noti anche come "antibiogrammi cumulativi", sono strumenti utilizzabili con finalità clinicoepidemiologiche (4). Il laboratorio dovrebbe fornire questi report sia come supporto per la scelta empirica degli antimicrobici, sia per l'aggiustamento dei protocolli terapeutici (9). Tale applicazione è problematica per quei microrganismi a basso impatto epidemiologico oppure difficilmente rilevabili in coltura come gli anaerobi. Per microrganismi $<30$ casianno è comunque accettabile la valutazione in un periodo maggiore, purché ciò sia chiaramente evidenziato (8).

Scopo del presente lavoro è la costruzione di una tabella locale per la terapia empirica. La tabella è indirizzata principalmente all'area chirurgica, con indicazioni epidemiologiche minime sulla flora anaerobica ricorrente e, soprattutto, con le indicazioni di suscettibilità (antibiogramma cumulativo). È stato previsto che l'implementazione della tabella sia subordinata alla verifica di congruità dei dati locali con i riferimenti della letteratura.

\section{MATERIALI E METODI}

Abbiamo effettuato uno studio di tipo descrittivo-retrospettivo del periodo luglio 2001 / giugno 2009 presso l'Azienda Ospedaliera San Filippo Neri di Roma, un ospedale a vocazione chirurgica ( $\sim 700$ posti letto nel periodo di studio) con un'elevata movimentazione di campioni per esame microbiologico, principalmente provenienti dal distretto addominale di pazienti ricoverati in regime ordinario (elezione ed urgenza), come Day Hospital o Day Surgery nell'intero ospedale. La fonte dati è stata il database di laboratorio (LIS).

Sono stati considerati isolati clinici unici, in accordo con il consensus del Clinical and Laboratory Standards Institute (CLSI M39-A2). I dati sono stati elaborati con il programma Mercurio (Noemalife). Sono state valutate le seguenti variabili: genere microbico, materiale biologico, metodologia di prelievo (tampone o liquido), reparto (area clinica), produzione di beta-lattamasi, suscettibilità $(\% \mathrm{~S})$ ad amoxicillinaacido clavulanico, cefoxitina, clindamicina, cloramfenicolo, imipenem, metronidazolo e piperacillina-tazobactam. Le suscettibilità dell'intero periodo sono state valutate in forma cumulativa.

I campioni sono stati messi in coltura su terreni solidi (Columbia, Chocolate, Schaedler KV) e liquidi: flaconi Bactec $^{\circledR}$ (Becton Dickinson, Franklin Lakes, NJ, USA) per anaerobi arricchiti con $\mathrm{FOS}^{\circledR}$ nel 2001-2004 e Thioglycollate Medium pre ridotto dal 2005 in poi [peraltro, con significativo incremento della quota di anaerobi recuperati, rispetto ai flaconi per emocoltura (1)]. Identificazioni ed antibiogrammi sono stati effettuati mediante Rapid ID32A ${ }^{\circledR}$ e ATB-ANA (bioMérieux, Marcy-L'Etoile, France) (5). Non è stato effettuato, continuativamente, un controllo di qualità specifico per microrganismi anaerobi; nel 2009 il laboratorio ha partecipato al "Anaerobe Survey" del UK-Neqas, con totale aderenza ai risultati attesi (sia per identificazione che per antibiogramma); dal 2001 aderisce peraltro alla VEQ "General Bacteriology" di UK-Neqas.

\section{RISULTATI}

Sono stati valutati 255 isolati unici: 41 Gram positivi (ripartiti su 9 generi) e 214 Gram negativi (ripartiti su 4 generi). Bacteroides e Prevotella sono stati i generi più rappresentati: 143 e 46 ceppi, rispettivamente. I microrganismi sono stati recuperati in $82 \%$ dei casi $(95 \% \mathrm{CI}$ : $78-87)$ direttamente dal sito chirurgico, mediante aspirazione di materiale liquido, prelievo di frammenti tissutali o con tampone. La ricerca su sangue (emocoltura) è stata utile nel $18 \%(95 \% \mathrm{CI}$ : 13-23) dei casi. L'uso dei tamponi (con terreno di trasporto), purtroppo ancora molto diffuso, non sembra comunque aver giocato un ruolo del tutto negativo nel recupero di microrganismi anaerobi. Il 35\% (95\%CI: $30-40)$ proveniva infatti da pus prelevato

\section{Corresponding author: Marcello Meledandri}

UOC Microbiologia e Virologia - Dipartimento di Scienze Radiologiche e Medicina di Laboratorio - AO San Filippo Neri

Via Martinotti 20,00I35 Roma - Tel.: 0633062650 - Fax: 06 330623I4

E-mail:m.meledandri@sanfilipponeri.roma.it 
con tale metodica, contro il 43\% (95\%CI: $37-49)$ degli isolati provenienti da liquidi aspirati. La quota maggiore, $(65 \%$; $95 \% \mathrm{CI}$ : 59-71) dei microrganismi era attribuibile all'area chirurgica.

La Tabella 1 riassume gli isolamenti microbici cumulativi dell'intero periodo e la suscettibilità agli antimicrobici $(\% \mathrm{~S}$ per genere e per raggruppamento; la percentuale non è visualizzata per isolati $<30$ casi). Tale tabella presenta, nella sua parte destra, il cosiddetto "antibiogramma cumulativo" locale, in teoria utilizzabile per regolare la terapia empirica.

I dati in tabella mostrano una frequenza globale di ceppi betalattamasi produttori pari all' $81 \%(95 \% \mathrm{CI}$ : $76-86)$ con un valore intorno al 90\% (95\%CI: 86-94) nei Gram negativi.

Le molecole che, in vitro risultano essere più efficaci verso il bio-burden complessivo degli anaerobi $(>95 \% \mathrm{~S})$, sono risultate: cloramfenicolo, imipenem, metronidazolo e piperacillina-tazobactam. Appare comunque buona $(80-90 \%$ S) la suscettibilità ad amoxicillina-clavulanato e cefoxitina, mentre la clindamicina mostra una efficacia complessivamente mediocre $(\sim 64 \% \mathrm{~S})$; ancora più evidente $(<63 \% \mathrm{~S})$ se si considera il raggruppamento dei Gram negativi.

\section{DISCUSSIONE}

Negli ultimi venti anni, si è assistito a un rilevante aumento della resistenza antibiotica in diverse specie di anaerobi anche se, dall'altra parte, non c'è evidenza di ricadute di questo fenomeno sull'outcome clinico (15). Tuttavia, molti microrganismi anaerobi Gram negativi possono mostrare resistenze non prevedibili, in grado di indurre terapie inappropriate (18). Inoltre, la natura polimicrobica di queste infezioni e il frequente uso di drenaggi, come accade in chirurgia gastrointestinale, possono oscurare i patogeni resistenti. Uno studio multicentrico prospettico di batteriemie da Bacteroides spp. ha mostrato un outcome peggiore, con persistenza batteriologica, nel gruppo con terapia inefficace rispetto a quello con la terapia efficace (13). E noto, infine, che la presenza degli anaerobi è in grado di modificare in maniera significativa sia la natura della peritonite (primitiva vs secondaria), sia la prognosi in termini di shock settico (32\% vs 9\%; odds ratio 2.78 ; $95 \%$ CI $1.07-7.21)$ e di mortalità (RR 0.88; 95\%CI 0.33-2.35) $(2,14,4)$.

La ricerca di routine dei batteri anaerobi è difficoltosa, ma è l'unico modo per studiare l'epidemiologia; recenti linee guida hanno riconosciuto un ruolo all'indagine microbiologica, oltre che nelle patologie complesse, proprio nello studio dell'epidemiologia locale (16).

L'epidemiologia locale ha rivelato un assetto microbico dominato da bacilli anaerobi Gram negativi. La comparazione dei dati con il survey multicentrico belga (18) ha evidenziato una buona concordanza in termini di suscettibilità.

La frequenza di beta-lattamasi nei batteri Gram negativi è pari a $90 \%$, contro il $98 \%$ dello studio belga. La clindamicina, come nello studio belga, ha una sensibilità globale del 64.5\%: questo dato non supporta l'uso di tale molecola, in empirica, nelle infezioni profonde. Tra i beta lattamici saggiati, la maggiore attività è stata riscontrata in piperacillinatazobactam $(97 \% \mathrm{~S})$, contro $87 \%$ di amoxicillina-clavulanato. Rispetto allo studio belga, la sensibilità di amoxicillinaclavulanato è più bassa $(87 \%$ vs $92 \%)$ : questo dato potrebbe essere dovuto al notevole uso di questo farmaco in empirica $\mathrm{e}$, in alcuni casi, in profilassi (peraltro, questo farmaco è stato inserito come prima scelta nella recente revisione dei protocolli locali terapia di empirica in chirurgia addominale).

Il Cloramfenicolo sembra aver conservato un'attività eccellente, così come metronidazolo (la cui resistenza rimane un evento eccezionale specialmente tra anaerobi Gram negativi). La quasi totalità degli isolati è risultata sensibile a imipenem. Complessivamente, questo studio ha permesso di documentare come la suscettibilità locale dei patogeni anaerobi non sia "ovvia e prevedibile". Pertanto, appare evidente che l'identificazione e l'antibiogramma sono imprescindibili, specialmente nelle infezioni complicate. Il pattern di suscettibilità riscontrato è apparso in accordo con i dati provenienti da raccolte di ceppi su larga scala (18): per questa ragione, i dati in tabella sono stati utilizzati per la stesura dei protocolli di terapia empirica in area chirurgica.

Tabella I. Tabella epidemiologica locale ed antibiogramma cumulativo dei microrganismi anaerobi

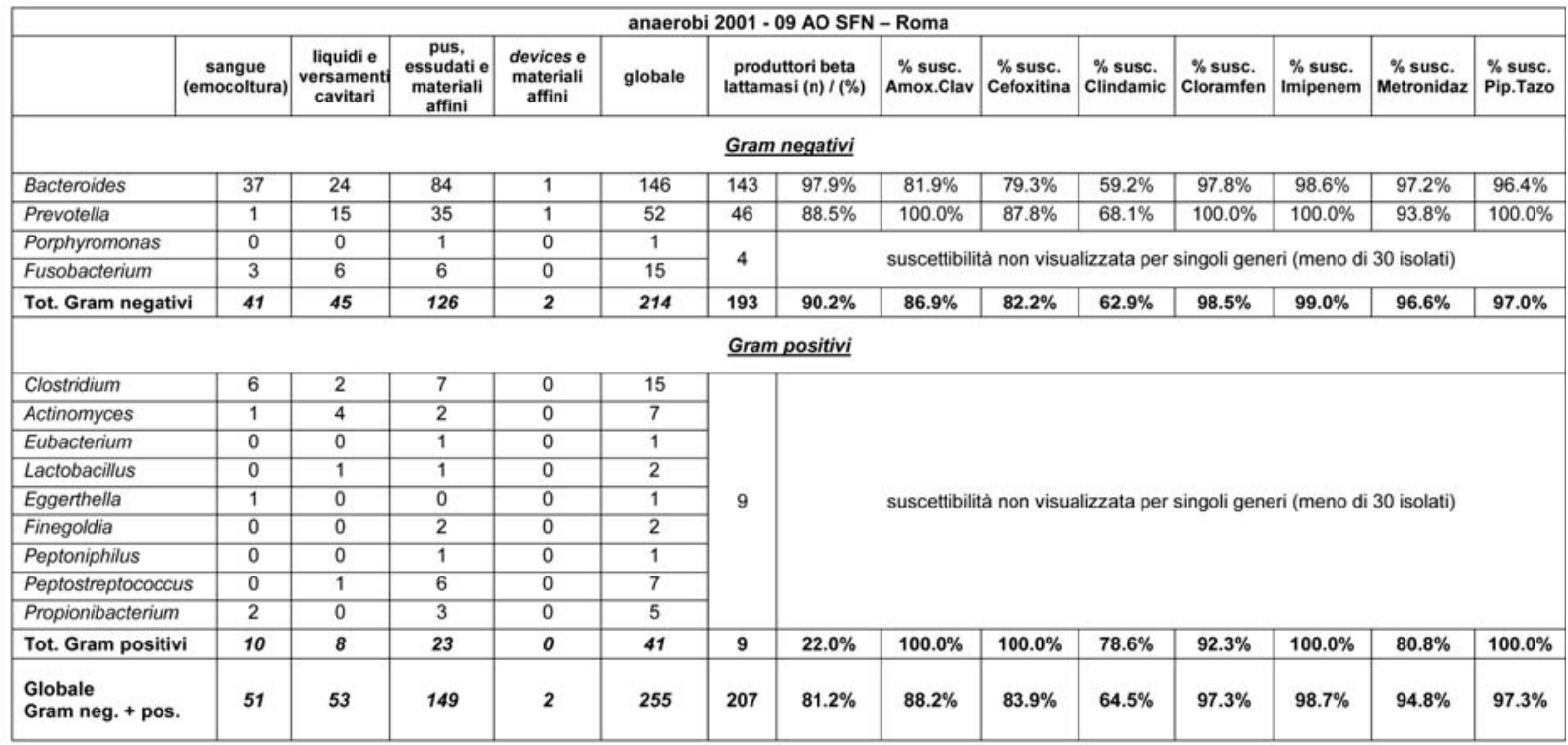

\section{BIBLIOGRAFIA}

1. Ballardini M, et al. Ricerca degli anaerobi: una modifica alla metodica "tradizionale" può cambiare l'epidemiologia? Esperienza locale. XXXVII Congresso Nazionale AMCLI. Abstract Book. Poster Session. 2008; N004

2. Bozovich ME, Marcello PW. Intra-abdominal Sepsis. In O’Donnell JM, Nácul FE (Eds). Surgical Intensive Care Medicine $2^{\text {nd }}$ ed., Springer, 2010

3. Brook I. Microbiology and Management of Abdominal Infections. Dig Dis Sci 2008; 53: 2585-91.
4. Clinical and Laboratory Standards Institute (CLSI). Analysis and presentation of cumulative antimicrobial susceptibility test data. $2^{\text {nd }}$ ed. Approved guideline M39-A2. Wayne, PA: CLSI, 2006.

5. Dubreuil L, Houcke I, Singer E. Susceptibility Testing on Anaerobic Bacteria: Evaluation of the Redesigned bioMérieux ATB ANA Device. Journal of Clinical Microbiology. 1999; 37: 1824-8.

6. Edmiston CE, et al. Anaerobic Surgical Infections, CID 2002; 35 (Suppl 1): S112-8.

7. Hasper D, et al. Management of Severe Abdominal Infections. Recent Pat 
Antiinfect Drug Discov. 2009; 4: 57-65.

8. Hindler JF, Stelling J. Analysis and Presentation of Cumulative Antibiograms: A New Consensus Guideline from the Clinical and Laboratory Standards Institute. Clinical Infectious Diseases 2007; 44: 867-73.

9. Jenkins SG. Quality Assurance. In Isemberg HD. Clinical Microbiology Procedures Handbook. $2^{\text {nd }}$ ed. Volume 3. Wahington, DC, ASM Press 2004; 14: $1-9$

10. Mangels JI. Anaerobic Bacteriology. In Isemberg HD. Clinical Microbiology Procedures Handbook. $2^{\text {nd }}$ ed. Volume 1. Wahington, DC: ASM Press 2004: Section 4.

11. Mazuski JE, Solomkin JS. Intra-abdominal infections. Surg Clin North Am. 2009; 89: 421-37.

12. Mazuski JE. Antimicrobial treatment for intra-abdominal infections. Expert Opin Pharmacother. 2007; 8: 2933-45.

13. Nguyen $\mathrm{MH}$, et al. Antimicrobial resistance and clinical outcome of
Bacteroides bacteraemia: findings of multicenter prospective observational trial. Clin Infect Dis 2000; 30: 870-6

14. Riché FC, Dray X, Laisné M-J, et al. Factors associated with septic shock and mortality in generalized peritonitis: comparison between communityacquired and postoperative peritonitis. Crit Care 2009; 13: R99.

15. Salonen JH, et al. Clinical significance and outcome of anaerobic bacteremia. Clin Infect Dis 1998; 26: 1413-7.

16. Solomkin JS, et al. Complicated Intra-abdominal Infection Guidelines. CID 2010; 50 (15): 133-64

17. Solomon JP, Nichols RL. Intrabdominal Infections. In: Cunha BA (eds) Infections Diseases in Critical Care Medicine. Marcel Dekker, New York, 1988: 461-88.

18. Wybo I, et al. Third Belgian multicentre survey of antibiotic susceptibility of anaerobic bacteria. Journal of Antimicrobial Chemotherapy 2007; 59: $132-9$ 